\title{
Aus der Forschung ins Pflegestärkungsgesetz II: Das Projekt Reha XI zur Feststellung des Rehabilitationsbedarfs in der MDK-Pflege- begutachtung
}

\author{
From Science to Law: Findings of Reha XI Project on Ascertaining \\ the Need for Rehabilitation in Medical Service Assessments
}

\section{(c) (1) $\circledast$}

\section{Autoren}

T. Kalwitzki ${ }^{1}$, K. Huter ${ }^{1}$, R. Runte ${ }^{1}$, K. Breuninger ${ }^{2}$, S. Janatzek²,

S. Gronemeyer ${ }^{2}$, B. Gansweid ${ }^{3}$, H. Rothgang ${ }^{1}$

Institute

1 SOCIUM - Forschungszentrum Ungleichheit und Sozialpolitik, Universität Bremen, Bremen

2 MDS, Essen

3 Leiterin des Fachreferates Pflege, Medizinischer Dienst der Krankenversicherung Westfalen-Lippe, Leiterin der SEG 2

„Pflege“, Bielefeld

\section{Schlüsselwörter}

medizinische Rehabilitation, Pflegebegutachtung, Rehabilitationsbedarf, Gute-Praxis-Standard, Evaluation, Pflegebedürftigkeit

\section{Key words}

medical rehabilitation, long-term care assessment, need for rehabilitation, good practice standard, evaluation, need for care

\author{
Bibliografie \\ DOI http://dx.doi.org/10.1055/s-0042-103745 \\ Online-Publikation: 2.5.2016 | Gesundheitswesen 2017; 79: \\ e26-e38 \\ (c) Georg Thieme Verlag KG Stuttgart · New York \\ ISSN 0941-3790
}

\section{Korrespondenzadresse}

Thomas Kalwitzki, Diplom-Gerontologe

SOCIUM - Forschungszentrum Ungleichheit und Sozialpolitik

Universität Bremen

Mary-Somerville-Straße 3

28359 Bremen

thomas.kalwitzki@uni-bremen.de

\section{ZUSAMMENFASSUNG}

Einleitung In dem breit angelegten Verbundprojekt „Reha XI - Erkennung rehabilitativer Bedarfe in der Pflegebegutachtung des MDK; Evaluation und Umsetzung “ wurde auf Basis einer umfassenden Ist-Analyse der Verfahrensabläufe ein Gute-Praxis-Standard (GPS) für die Begutachtung entwickelt und wissenschaftlich evaluiert. Der Artikel diskutiert die Ergebnisse und die Anwendbarkeit des GPS als Grundlage eines bundeseinheitlichen Verfahrens, wie es durch das Zweite Pflegestärkungsgesetz in $§ 18$ Abs. 6 SGB XI vorgesehen ist.

Methode Das Projekt wurde als wissenschaftliches Verbundprojekt in
4 Projektschritten durchgeführt: 1. Ist-Analyse der Prozesse bei der Feststellung des Rehabilitationsbedarfs in der MDK-Pflegebegutachtung; 2. Entwicklung eines GPS in einem strukturierten Konsensverfahren; 3. wissenschaftliche Evaluation der Validität, Reliabilität und Praktikabilität des Begutachtungsablaufs nach GPS innerhalb des Praxisbetriebes der MDK; 4. Befragung der Pflegekassen zur Eignung der nach GPS erstellten Rehabilitationsempfehlungen im Pflegegutachten zur Übermittlung einer qualifizierten Empfehlung an die Antragsteller. Die im dritten Projektschritt durchgeführte Evaluation war mit methodischen Limitationen behaftet, die Verzerrungen der Evaluationsergebnisse möglich erscheinen lassen.

Ergebnisse Auf Basis der Ist-Analyse konnten 7 wesentliche Themenbereiche identifiziert werden, in denen über den GPS Prozessverbesserungen implementiert wurden. Im Rahmen seiner Evaluation wurden 3247 Antragsteller nach GPS begutachtet, bei 6,3\% dieser Antragsteller wurde eine Indikation für eine Leistung der medizinischen Rehabilitation festgestellt. Für die Vorgehensweise nach GPS zeigte sich eine gute Reliabilität und Praktikabilität, während für die Validität des Begutachtungsablaufs nur sehr unbefriedigende Werte ermittelt wurden. Die Akzeptanz der nach GPS erstellten Rehabilitationsempfehlungen bei den Pflegekassen war gut.

Schlussfolgerung Die MDK-übergreifende Anwendung eines Standards für dieses Begutachtungsfeld bringt deutliche Qualitätsverbesserungen des Begutachtungsablaufs und führt häufiger zur Feststellung einer Indikation zur medizinischen Rehabilitation. Die methodischen Probleme und die unbefriedigenden Ergebnisse hinsichtlich der Validität der gutachtlichen Entscheidungen bedürfen einer weiteren wissenschaftlichen Bearbeitung.

\section{ABSTRACT}

Introduction In the broad-based consortium project "Reha XI - Identifying rehabilitative requirements in medical service assessments: evaluation and implementation", a comprehensive analysis of the corresponding procedures was carried out by the medical services of the German Health Insurance Funds (MDK). On the basis of this analysis, a Good Practice Standard (GPS) for assessments was drawn up and scientifically evaluated. This article discusses the findings and applicability of the GPS as the basis for a nationwide standardized procedure in Germany as required by the Second Act to Strengthen Long-Term Care (PSG II) under Vol. XI Para. 18 (6) of the German Social Welfare Code.

Method The consortium project comprised four project phases: 1. Qualitative and quantitative situation analysis of the procedures for ascertaining rehabilitative needs in care assessments carried out by the MDK; 2. Development of a Good Practice Standard (GPS) in a structured, consensus-based procedure; 3 . Scientific evaluation of the validity, reliability and practicability of the assessment procedure according to the 
GPS in the MDK's operational practice; 4. Survey of long-term care insurance funds with respect to the appropriateness of the rehabilitation recommendations drawn up by care assessors in line with the GPS for providing a qualified recommendation for the applicant. The evaluation carried out in the third project phase was subject to methodological limitations that may have given rise to distortions in the findings.

Findings On the basis of the situation analysis, 7 major thematic areas were identified in which improvements were implemented by applying the GPS. For the evaluation of the GPS, a total of 3247 applicants were assessed in line with the GPS; in $6.3 \%$ of the applicants, an indication for medical rehabilitation was determined. The GPS procedure showed a high degree of reliability and practicability, but the values for the validity of the assessment procedure were highly unsatisfactory. The degree of acceptance by the long-term care insurance funds with respect to the recommendations for rehabilitation following the GPS procedure was high.

Conclusion The application of a general standard across all MDKs shows marked improvements in the quality of the assessment procedure and leads more frequently to the ascertainment of an indication for medical rehabilitation. The methodological problems and the unsatisfactory findings with respect to the validity of the assessors' decisions require further scientific scrutiny.

\section{Einleitung}

Das am 1.1.2016 in Kraft getretene Zweite Pflegestärkungsgesetz (PSG II) sieht unter anderem vor, dass die Feststellungen zum Rehabilitationsbedarf im Rahmen der Pflegebegutachtung künftig auf der Grundlage eines bundeseinheitlichen, strukturierten Verfahrens zu treffen sind. In der Begründung zu dieser Vorschrift nimmt der Gesetzgeber Bezug auf das 2014 abgeschlossene wissenschaftliche Projekt Reha XI, in dem dieses bundeseinheitliche, strukturierte Verfahren entwickelt und evaluiert wurde. Da die Bedeutung dieses Verfahrens durch seine Verankerung im Recht der Sozialen Pflegeversicherung nun hervorgehoben wird, werden in der folgenden Übersicht die Ziele, Methoden und Ergebnisse des Projektes Reha XI erstmals ausführlich publiziert.

Leistungen zur medizinischen Rehabilitation haben zum Ziel, eine Behinderung einschließlich Pflegebedürftigkeit abzuwenden, zu mindern oder eine Verschlimmerung zu verhüten (§ 4 SGB IX, $\S+11$ SGB V). Der Grundsatz „Rehabilitation vor Pflege“ ist an verschiedenen Stellen im deutschen Sozialversicherungssystem gesetzlich verankert.

Klare Vorgaben zur Umsetzung dieses Grundsatzes enthält unter anderem das Sozialgesetzbuch - Elftes Buch - Soziale Pflegeversicherung (SGB XI). Danach ist gemäß §+18 SGB XI, bei jeder Pflegebegutachtung auch zu prüfen, ob und in welchem Umfang Maßnahmen zur Beseitigung, Minderung oder Verhütung einer Verschlimmerung der Pflegebedürftigkeit notwendig und zumutbar sind. Dies schließt Leistungen zur medizinischen Rehabilitation ausdrücklich ein.

An der Umsetzung dieses gesetzlichen Auftrages durch die Medizinischen Dienste der Krankenversicherung (MDK) und die Pflegekassen wurde in der Vergangenheit häufig Kritik geübt, da es lediglich bei weit unter einem Prozent der vom MDK begutachteten pflegebedürftigen Personen zu einem Antrag auf Leistungen zur medizinischen Rehabilitation kommt [1]. Inwieweit diese Begutachtungsergebnisse eine angemessene Prüfpraxis widerspiegeln, ließ sich bislang kaum nachvollziehbar beurteilen, da empirische Erkenntnisse zum Rehabilitationsbedarf Pflegebedürftiger weitgehend fehlen. Unabhängig davon, haben die anhaltend niedrigen Empfehlungsquoten in der MDK-Pflegebegutachtung den Gesetzgeber veranlasst, in den Pflegereformen der letzten Jahre (PflegeWeiterentwicklungsgesetz, PfWG, 2008, Pflege-Neuausrichtungsgesetz, PNG, 2012) immer wieder neue Vorschriften zu erlassen, die auf eine stringentere Umsetzung des Grundsatzes „Reha vor Pflege “ abzielten. Allerdings blieb eine erkennbare Wirkung dieser gesetzlichen Vorgaben bislang weitgehend aus.
Die Feststellung einer Indikation zur medizinischen Rehabilitation im Rahmen der Pflegebegutachtung durch den MDK gilt als Antrag nach §+ 14 SGB IX, und die Versicherten haben einen Anspruch auf eine ausführliche gesonderte Rehabilitationsempfehlung, die ihnen von der Pflegekasse mit dem Leistungsbescheid zu übermitteln ist.

Vor dem Hintergrund der erwähnten Kritik an MDK und Pflegekassen wurde das Projekt „Reha XI - Erkennung rehabilitativer Bedarfe in der Pflegebegutachtung des MDK; Evaluation und Umsetzung “ (kurz: Reha XI) initiiert. Aufgabe des Projektes war es, die Transparenz der Vorgehensweise der MDK in Bezug auf diesen Teil des Pflegebegutachtungsprozesses zu erhöhen, Stärken und Schwächen des Verfahrens zu analysieren und auf dieser Basis einen übergreifenden Gute-Praxis-Standard (GPS) für die MDK-Gemeinschaft zu entwickeln und anschließend zu evaluieren. Ziel dieses Verbundprojektes war damit einerseits eine Qualitätsentwicklung innerhalb der MDK, andererseits wurde auch eine Grundlage geschaffen, um die vorgetragene Kritik auf wissenschaftlicher Basis zu versachlichen.

An der Durchführung des Projektes waren der GKV-Spitzenverband, der Medizinische Dienst des Spitzenverbandes Bund der Krankenkassen (MDS), Experten aus den Verbänden der Kranken- und Pflegekassen auf Bundesebene und der Medizinischen Dienste der Krankenversicherung (MDK) beteiligt. Die wissenschaftliche Begleitung erfolgte durch das Zentrum für Sozialpolitik (ZeS) ${ }^{1}$ der Universität Bremen. Das Projekt hatte eine Laufzeit von 13 Monaten.

\section{Hintergrund, Problemstellung und Ziele des Projektes}

Der Zugang zu Leistungen der medizinischen Rehabilitation der GKV erfolgt zum einen auf Veranlassung von Krankenhausärzten im Anschluss an eine Krankenhausbehandlung als Anschlussrehabilitation, zum anderen durch die Verordnung von Vertragsärzten aus dem ambulanten Bereich gemäß Rehabilitations-Richtlinie des Gemeinsamen Bundesausschusses. Neben diesen Zugangswegen sind die Kranken- und Pflegekassen sowie der MDK verpflichtet, auf die Einleitung präventiver und rehabilitativer Maßnahmen hinzuwirken, auch und gerade wenn Pflegebedürftigkeit unmittelbar droht oder bereits eingetreten ist.

1Jetzt SOCIUM - Forschungszentrum Ungleichheit und Sozialpolitik 
Daher ist gesetzlich vorgeschrieben, dass die Gutachter des MDK im Rahmen der Begutachtung von Pflegebedürftigkeit auch feststellen, ob und in welchem Umfang Maßnahmen zur Beseitigung, Minderung oder Verhütung einer Verschlimmerung der Pflegebedürftigkeit einschließlich Leistungen zur medizinischen Rehabilitation geeignet, notwendig und zumutbar sind. Die MDK-Gemeinschaft hat dazu ein 2-stufiges Verfahren etabliert, das bei der Indikationsstellung zu Leistungen der medizinischen Rehabilitation eine strukturierte Zusammenarbeit von pflegefachlichen und ärztlichen MDK-Gutachtern vorsieht. Dabei haben die in der Pflegebegutachtung eingesetzten Pflegefachkräfte die Aufgabe, auf Basis des Hausbesuchs Hinweise auf das mögliche Vorliegen einer Rehabilitations-Indikation zu erfassen. Werden solche Hinweise festgestellt, wird eine endgültige Entscheidung über die RehabilitationsIndikation durch einen zu diesem Zweck hinzugezogenen ärztlichen Gutachter getroffen. Damit wird sichergestellt, dass die endgültige Indikationsstellung zur medizinischen Rehabilitation eine ärztliche Entscheidung ist. Wie diese für die MDK verbindlich vorgegebene Vorgehensweise im Detail umgesetzt wird, welche Probleme dabei möglicherweise bestehen und ob damit Personen mit Rehabilitations-Indikation zuverlässig ermittelt werden, wurde bisher nicht systematisch untersucht.

Bezüglich der Umsetzung des Prüfauftrages durch die MDK werden die bisherigen Anteile festgestellter Rehabilitations-Indikationen zum Teil als zu niedrig kritisiert (z. B. [2, 3]). Im Hinblick auf diese Kritik ist jedoch zu beachten, dass es bisher keine empirisch abgesicherten Erkenntnisse zur Höhe des Bedarfs an Leistungen zur medizinischen Rehabilitation bei Antragstellern der Pflegeversicherung gibt und die Fokussierung auf komplexe Leistungen der medizinischen Rehabilitation dazu führt, Empfehlungen der MDK zu Einzelleistungen mit rehabilitativer Zielsetzung wie Heil- und Hilfsmittel zu marginalisieren. Darüber hinaus ist jeweils die individuelle Fallkonstellation des Antragstellers zu berücksichtigen. Wenn z. B. gerade erst eine Rehabilitationsleistung erfolgt ist (vgl. [4]) oder der Antragsteller eine Rehabilitationsmaßnahme ausdrücklich ablehnt, sind die Voraussetzungen für eine Rehabilitations-Indikation nicht gegeben.

Grundsätzlich befindet sich der MDK-Gutachter eher am Ende einer Versorgungskette, weil das Vorliegen einer RehabilitationsIndikation in der Regel schon vom Hausarzt oder im Rahmen einer Krankenhausbehandlung abgeklärt sein sollte. Anderseits hat der MDK-Gutachter aber die Möglichkeit, Personen mit einer Rehabilitations-Indikation zu identifizieren, die von den anderen Akteuren nicht als solche erkannt wurden, also quasi „durch das Netz gerutscht" sind.

Über diese prozessualen Aspekte hinaus, existiert eine Erkenntnislücke in Bezug auf die inhaltlichen Aspekte der Feststellung einer Rehabilitations-Indikation. Nach dem SGB XI hat sich der Umfang der zu empfehlenden Leistungen mit rehabilitativer Zielsetzung daran zu orientieren, inwieweit das in Frage kommende Leistungsangebot geeignet, notwendig und zumutbar ist.

Dies setzt Erkenntnisse darüber voraus, welche Angebote bei welchem Versicherten tatsächlich zu einer Vermeidung, Verminderung oder Verhütung einer Verschlimmerung von Pflegebedürftigkeit führen können. Bestehen bezüglich der Wirksamkeit und des Nutzens geriatrischer Rehabilitationen noch „moderate Evidenzen“, fehlen diese nahezu völlig zu der Frage, wie lange, in welcher Form, durch wen und in welcher Intensität rehabilitative Maßnahmen bei erheblich beeinträchtigten (alten) Menschen gegenüber deutscher Standardversorgung bspw. im Sinne einer aktivierend-pflegerischen Grundversorgung wirksam und nutzbringend sind (vgl. [5]).

Gesamtziel des Projektes war es, die Umsetzung der gesetzlich vorgeschriebenen Aufgaben zur Erkennung rehabilitativer Bedarfsaspekte in der Pflegebegutachtung der MDK transparenter zu machen, Schwachstellen zu identifizieren und eine Grundlage für notwendige Qualitätsverbesserungen zu schaffen. Insbesondere wurde auf Basis einer Ist-Analyse ein Gute-Praxis-Standard (GPS) entwickelt, der auf einer Reihe von optimierten Prozessbausteinen aufbaut und durch die beteiligten Experten bewertet, fachlich diskutiert und einvernehmlich konsentiert wurde. Der resultierende Handlungsleitfaden wurde anschließend in einigen MDK implementiert, in der Praxis erprobt und dabei evaluiert.

\section{Methodik}

\section{Grundansatz}

Sowohl bei der Planung als auch bei der Durchführung des Projektes wurden alle beteiligten Personen und Hierarchieebenen sowie die MDK-Gemeinschaft in ihrer dezentralen Struktur in die Generierung der empirischen Erkenntnisse einbezogen. Dieses partizipative Vorgehen zog sich durch alle Projektphasen und ermöglichte es, den Wissensgewinn mit einer konsequenten Bottom-up-Struktur offen zu verfolgen und die daraus folgenden Impulse für die Organisationsentwicklung auf einer breiten Basis zu konsentieren.

\section{Ist-Analyse}

Als Untersuchungsdesign für die Bestandsaufnahme wurde ein 3-schrittiges Vorgehen aus qualitativen und quantitativen Elementen gewählt, um eine möglichst umfassende Perspektive auf den Prozess - sowohl im Detail als auch in der Fläche - zu erhalten.

In einem ersten Projektschritt wurden qualitative Experteninterviews mit Vertretern des MDS und der SEG 2 (Sozialmedizinische Expertengruppe der MDK-Gemeinschaft Pflege/Hilfebedarf) sowie mit 12 Mitarbeitern verschiedener Funktionen aus 3 ausgewählten MDK geführt. Mit diesen Interviews sollte ein möglichst differenziertes Bild der Prozessabläufe im Begutachtungsprozess gewonnen werden, um Ansatzpunkte für die Identifikation von Problembereichen und regionalen Unterschieden zu gewinnen. Auf diesen Ergebnissen aufbauend wurde eine Online-Befragung durchgeführt. An dieser beteiligten sich 14 MDK mit insgesamt 144 Personen verschiedener Funktionen. Ergänzend wurden durch einen Fragebogen Strukturdaten der einzelnen MDK (z. B. zur Anzahl der Beschäftigten und den durchgeführten Pflegebegutachtungen) erhoben, die als Hintergrundinformationen genutzt wurden.

Um die Ergebnisse um eine über die individuelle Perspektive hinausgehende Ebene zu ergänzen, wurden anschließend 6 Gruppendiskussionen in 4 MDK durchgeführt. In diesen diskutierten ärztliche und pflegefachliche Gutachter entweder berufsgruppenhomogen oder in gemischten Settings die Kernergebnisse der vorangegangenen Erhebungsphasen. Diese ermöglichten einen gezielten Einblick in die realen Lösungs- und Begründungsstrukturen vor Ort, deren argumentative Hintergründe im Gespräch offengelegt wurden. Inhalte der Gruppendiskussionen waren insbesonde- 
re die Qualifikation und der Schulungsbedarf der pflegefachlichen Gutachter, Einstellungen und Reha-Verständnis ärztlicher und pflegefachlicher Gutachter, in den Prozessabläufen auftretende Schwierigkeiten sowie die Kommunikations- und Kooperationskonflikte mit anderen Berufsgruppen.

\section{Entwicklung Gute-Praxis-Standard (GPS)}

Als erstes Zwischenergebnis zielte das Projekt Reha XI auf die Ausarbeitung, Konsentierung und Verabschiedung eines Gute-PraxisStandards (GPS) für den Prozess zur Feststellung der Rehabilitations-Indikationen im Rahmen der Pflegebegutachtung des MDK. GPS bezeichnet dabei eine Struktur- und Prozessbeschreibung, die im Wesentlichen aus praktisch erprobten Regelungen zusammengesetzt ist und damit die übergreifenden Anforderungen an den Begutachtungsprozess definiert.

Wichtig festzuhalten ist dabei, dass ein GPS im Unterschied zu einem Best-Practice-Standard nicht auf die Findung einer einzigen, besten Lösung ausgerichtet ist. Ziel ist es vielmehr, in ihrer Praktikabilität erprobte Regelungen zu finden, die mit den bestehenden Organisationszielen und -strukturen weitgehend kompatibel sind und dadurch einen nachhaltigen Erfolg versprechen. Gerade in Bezug auf die MDK-Gemeinschaft stellt sich diese Zielrichtung als viel versprechend dar.

Die Entwicklung des GPS wurde in 2 Schritten durchgeführt. Als ein Ergebnis der Ist-Analyse waren 9 Themenbereiche identifiziert worden, in denen Prozessverbesserungen erreicht werden sollten. Den Themenbereichen zugeordnet wurden 34 Praxisregelungen, die in einzelnen MDK bereits eingesetzt oder von MDK-Mitarbeitern als wünschenswerte Verfahrensänderungen oder -ergänzungen benannt worden waren. Diese empirisch erarbeiteten Vorschläge wurden in einem ersten Schritt in Form einer Online-Befragung einer Expertengruppe präsentiert und durch diese beurteilt. Dabei wurde sowohl die Zustimmung zu den vorgeschlagenen Regelungen, als auch deren Wichtigkeit für den Prozess erhoben. Die Expertengruppe setzte sich aus insgesamt 151 Mitarbeitern aller MDK, Mitgliedern der Sozialmedizinischen Expertengruppen der MDK-Gemeinschaft „Leistungsbeurteilung und Teilhabe“ (SEG 1) und „Pflege“ (SEG 2), des Kompetenz-Centrums Geriatrie(KCG) des GKV-Spitzenverbandes und der MDK-Gemeinschaft sowie des MDS zusammen. Von diesen beantworteten 102 Personen den Fragebogen vollständig. Das entspricht einem Rücklauf von 67,5\%. Aus den positiv bewerteten Vorschlägen wurde ein kohärenter Standard erarbeitet.

Der derart erarbeitete Entwurf des GPS wurde anschließend in einer Sitzung des projektberatenden und -begleitenden Expertenkreises einer abschließenden inhaltlichen und organisatorischen Prüfung unterzogen. Dabei zeigte sich eine weitgehende Zustimmung zur vorgestellten Entwurfsversion. Der Gute-Praxis-Standard ist somit in einem transparenten Prozess basierend auf den Einschätzungen der Betroffenen erarbeitet worden.

\section{Evaluation des GPS-optimierten 2-stufigen Verfahrens}

Um zu einer ersten Einschätzung der Qualität der Erkennung rehabilitativer Bedarfe im Rahmen der Pflegebegutachtung im durch den GPS optimierten 2-stufigen Verfahren zu kommen, wurde die Vorgehensweise in einem dritten Projektschritt in einer 3-armigen Studie evaluiert. Überprüft werden sollte erstens, ob das Verfah- ren eine valide Identifikation von Rehabilitations-Indikationen gewährleistet (Validität), zweitens, ob es von den beteiligten pflegefachlichen und ärztlichen Gutachtern zuverlässig angewendet werden kann (Reliabilität) und drittens, ob es in Bezug auf qualifikatorische und arbeitsorganisatorische Rahmenbedingungen in den Arbeitsalltag der Gutachter eingebunden werden kann (Praktikabilität). Die Umsetzung erfolgte in 3 unabhängigen und parallelen Studienarmen.

Dabei ging es in Bezug auf die Validität explizit darum, das GPSoptimierte 2-stufige Verfahren mit einem „optimalen“ Referenzverfahren - und nicht mit dem Status quo ante - zu vergleichen. Der wesentliche Grund dafür war, dass bisher keine empirische Überprüfung des angewendeten Verfahrens vorgenommen worden war, die schon als Referenzpunkt hätte verwendet werden können. Eine Bewertung der Qualitätsentwicklung durch den GPS war aus diesem Grund nicht möglich. Es war erklärtes Ziel, erste Abschätzungen zu ermöglichen, aus denen wiederum die Generierung weiterer Hypothesen zur Entwicklung des Verfahrens möglich war. Grundlage für diese entdeckende Perspektive war, dass bisher kaum wissenschaftliche Evidenz zur Güte analoger Entscheidungsalgorithmen und Vergleichsstandards besteht, auf die das Projekt Reha XI hätte aufbauen können (vgl. u. a. [6]). Um überhaupt Aussagen über die Validität des GPS-optimierten Verfahrens treffen zu können, war es erforderlich ein Referenzverfahren zu bestimmen, das als Vergleichsmaßstab eingesetzt werden konnte. Mangels eines wissenschaftlich fundierten Standards wurde in der Projektplanung festgelegt, als beste Referenz ein Verfahren zu wählen, in dem die zur Feststellung des Rehabilitationsbedarfs bestqualifizierte Berufsgruppe auf Basis der größtmöglichen Informationsmenge ihre Entscheidung trifft. Hierzu wurde der Hausbesuch eines in der Rehabilitationsbegutachtung erfahrenen Arztes ausgewählt. Aussagen zur Validität des Verfahrens entstanden dementsprechend dadurch, dass die Prüfungsergebnisse des 2-stufigen Verfahrens nach GPS mit den Ergebnissen des gesetzten, einstufigen Referenzverfahrens verglichen wurden. Hierzu war es methodisch erforderlich, die Ergebnisse des Referenzverfahrens grundsätzlich als richtig anzunehmen, um statistische Beurteilungen überhaupt vornehmen zu können. Hierbei handelt es sich jedoch um eine Brückenannahme, die kritisch betrachtet werden muss. Eine absolute Beurteilung wäre erst möglich, wenn valide Aussagen auch über die Güte dieses Referenzverfahrens vorlägen.

In der praktischen Durchführung wurden insgesamt 221 Antragsteller, bei denen im 2-stufigen Verfahren nach GPS keine Rehabilitations-Indikation festgestellt wurde, und 43 Antragsteller, bei denen im 2-stufigen Verfahren nach GPS eine Rehabilitations-Indikation festgestellt wurde, durch einen in der Rehabilitationsbegutachtung erfahrenen Arzt in einem zweiten Hausbesuch erneut begutachtet. Durch den Vergleich der Ergebnisse wurden als Kenngrößen der Testqualität der Positive und der Negative Prädiktive Wert, sowie Sensitivität und Spezifität errechnet. Zusätzlich konnten über das rein dichotome Testergebnis hinaus auch tiefere Analysen angestellt werden, die sich auf Entscheidungs-Begründungen oder Allokationsempfehlungen bezogen, sofern diese Daten in der dreiseitigen Rehabilitations-Empfehlung des Formulargutachtens enthalten waren.

Die Beurteilung der Personenunabhängigkeit der Anwendung des Begutachtungsablaufs wurde als Kenngröße für die Reliabilität 
des Verfahrens eingesetzt. Dazu wurden wiederum Doppelbegutachtungen vorgenommen und die Testergebnisse auf Übereinstimmungen oder Unterscheide verglichen. Überprüft wurde jeweils die erste Stufe des 2-stufigen Verfahrens, es wurden also die Ergebnisse zweier voneinander unabhängiger pflegefachlicher Gutachter verglichen. Dabei wurden 83 Antragsteller einbezogen, bei denen im ersten Hausbesuch nach GPS keine Rehabilitations-Indikation vermutet wurde, und 9 Antragsteller bei denen im ersten Hausbesuch nach GPS eine Rehabilitations-Indikation vermutet wurde.

Zur Einschätzung der Praktikabilität wurde eine Online-Befragung mit allen beteiligten Gutachtern durchgeführt. Von diesen beteiligten sich mit 73 pflegefachlichen Gutachtern und 28 Ärzten jeweils $85 \%$ der Studienteilnehmer. Ihnen wurden insgesamt 11 Fragen gestellt, zu denen Beurteilungen abgegeben oder freitextliche Erfahrungen und Verbesserungen mitgeteilt werden konnten.

\section{Methodische Einschränkungen}

Die gewichtigste methodische Einschränkung betrifft die für die Validitätsprüfung notwendige Festlegung des Referenzverfahrens. Diese ist in jedem Fall einer solchen Qualitätsprüfung erforderlich, birgt im vorliegenden Fall aber 2 einander bedingende Probleme. Da es sich bei der Feststellung einer Rehabilitations-Indikation um ein Verfahren mit einem gewissen Ermessensspielraum handelt, kann nicht von einer absoluten und korrekten, personenunabhängigen Zuordnung ausgegangen werden. Dies hat zur Folge, dass der Validierung des Referenzverfahrens eine gesteigerte Bedeutung zukommt, da nur durch sie auch eine Einordnung der jeweiligen Vergleichsergebnisse möglich ist. Eine solche Validierung liegt für das hier gewählte Referenzverfahren nicht vor.

Weitere Einschränkungen betreffen Probleme bei der Umsetzung der Regelungen des GPS, die nicht in allen MDK einheitlich erfolgen konnten, ein nicht immer einheitlicher Umgang mit Antragstellern, die eine Rehabilitation für sich von vornherein ablehnten, nicht vollständig auszuschließenden Effekten der Selbstselektion der teilnehmenden Antragsteller sowie Probleme im Umgang mit dem Online-Erhebungsinstrument und der Dateneingabe. In der Summe beinhalten diese Einschränkungen ein schwer einzuschätzendes Verzerrungspotenzial. Die mögliche Bedeutung der methodischen Limitationen wird abschließend ausführlicher zu diskutieren sein.

\section{Befragung der Pflegekassen}

Im letzten Abschnitt des Gute-Praxis-Standards ist als Abschluss des Prozesses die Datenübermittlung an die Pflegekassen geregelt worden. Sie stellt für die MDK als Anwender des GPS die Schnittstelle zum Auftraggeber dar. Die dabei den Pflegekassen im Pflegegutachten zur Verfügung gestellten Daten sollten dazu geeignet sein, diese in die Lage zu versetzen, ihrer gesetzlichen Verpflichtung zur Erstellung einer individuell-begründeten RehabilitationsEmpfehlung entsprechen zu können.

Ziel des letzten Projektteils war es daher zu überprüfen, inwieweit die hierfür notwendigen Informationen im optimierten Verfahren aus Sicht der Pflegekassen übermittelt werden. Es ist jedoch festzuhalten, dass in Bezug auf die Datenübermittlung von den MDK an die Pflegekassen durch den GPS keine Änderungen vorgenommen wurden. Vertreter der Pflegekassen hatten sich bei der
Diskussion des GPS im Konsensus-Workshop explizit gegen eine Erweiterung der übermittelten Inhalte ausgesprochen.

Die Befragung der Pflegekassen wurde als Online-Erhebung umgesetzt. In ihr wurden 7 dichotom skalierte und einige offene Fragen an den jeweiligen Sachbearbeiter gerichtet, der diese auf einen konkret vorliegenden Fall bezogen beantworten sollte. Die Fragen bezogen sich ausschließlich auf die Nachvollziehbarkeit der übermittelten Daten, deren Konsistenz, Qualität und ihre Aussagekraft in Bezug auf die gestellten gesetzlichen Anforderungen. In die Studie eingegangen sind 149 Fälle, die von 26 verschiedenen Pflegekassen bearbeitet wurden.

\section{Ergebnisse Ist-Analyse}

Insgesamt zeigte sich, dass die strukturelle Organisation der 15 MDK sehr verschieden ist. Diese Heterogenität setzt den Möglichkeiten einer Vereinheitlichung der Abläufe in einigen Bereichen klare Grenzen. Einen nicht hintergehbaren Rahmen stellen zudem die gesetzlichen Rahmenbedingungen dar, in die das Verfahren eingebettet ist. Daraus resultierende Schwächen können durch Verfahrensänderungen im GPS zum Teil abgemildert, nicht jedoch vollständig aufgelöst werden.

\section{In der Ist-Analyse identifizierte Schwächen bei der Feststellung des Rehabilitationsbedarfs}

Durch die Stärkung des Grundsatzes „Reha vor Pflege“ und der im Rahmen der Pflegebegutachtung notwendigen Prüfung, ob eine Rehabilitations-Indikation vorliegt, kommt es bei der Begutachtung zu einer Überschneidung verschiedener Leistungsbereiche des SGB. Während der Versicherte einen Antrag auf Leistungen nach SGB XI stellt, wird gleichzeitig geprüft, ob Leistungsansprüche nach $\S 40$ SGB V vorliegen. Dies wirkt sich auf 3 Ebenen problematisch aus. Die von den pflegefachlichen Gutachtern erwarteten Einschätzungen können über die eigentlichen Ausbildungsinhalte der Pflegefachkräfte hinausgehen und in ärztliche Kompetenzbereiche eingreifen. In Folge stellt sich zum einen die Frage nach dem Umfang des Schulungsbedarfs der Pflegefachkräfte und der grundsätzlichen Qualität ihrer Einschätzungen, zum anderen führt dies regelmäßig zu Konflikten mit Haus- und Fachärzten, die einen Eingriff in ihren Kompetenzbereich und die Versorgung ihrer Patienten wahrnehmen. Drittens ist das Verfahren für die Antragsteller selbst aus diesem Grund wenig transparent. Ihnen ist im Vorfeld in der Regel nicht bekannt, dass im Rahmen der Pflegebegutachtung auch auf Rehabilitations-Bedarf geprüft wird. Da für die Versicherten der Antrag auf Pflegeleistungen der primäre Anlass der Begutachtung darstellt, befürchten viele in der Begutachtungssituation, dass sich die Zustimmung zu einer Rehabilitationsleistung negativ auf die zu erwartenden Ansprüche auf Pflegeleistungen auswirken könnte. Fehlende Motivation der Antragsteller wird entsprechend auch als ein häufiger Abbruchgrund der Prüfung der Rehabilitations-Indikation von Seiten der pflegefachlichen Gutachter benannt ( $\vee$ Abb. 1).

Durch die Einführung von Zusatzzahlungen im Pflege-Neuausrichtungsgesetz, die dann fällig werden, wenn dem Antragsteller nicht binnen einer 5-Wochen-Frist ein Leistungsbescheid zugestellt wird, haben sich die zeitlichen Anforderungen in der Pflegebegut- 


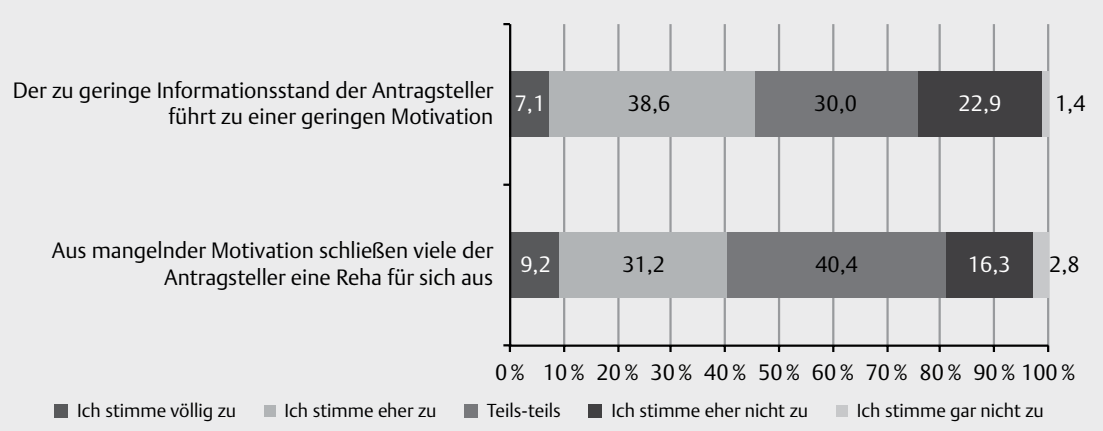

- Abb. 1 Folgen des geringen Informationsstandes der Antragsteller. Quelle: Eigene Darstellung nach den Daten der Online-Befragung.

achtung verändert. Dies wirkt - zumindest ist dies ein Ergebnis der Befragung der Gutachter - auf den Gesamtprozess sowohl auf organisatorischer als auch auf personeller Ebene ein. Danach werden die Begutachtungssituationen selber durch neue Aufgaben bei gleichem Zeitbudget als aufwendiger empfunden, aber auch die Verwaltung der Fälle erfolgt offenbar unter höherem Zeitdruck. Dies führe z. B. zu verringerten Möglichkeiten ergänzende Unterlagen anzufordern, wodurch die Gutachtenqualität sinken könne. Zusätzlich verringerten sich teilweise die Vor- und Nachbereitungszeiten für die Gutachter, da kurzfristige Veränderungen der Tourenplanung eine Vorbereitung der Fälle erschweren und längere Wege zwischen den einzelnen Antragstellern absolviert werden müssen.

Neben diesen Problemkonstellationen, die in direkter Beziehung zu den rechtlichen Rahmenbedingungen stehen, zeigten sich jedoch auch eine Reihe weiterer Ansatzpunkte für Qualitäts- und Verfahrensverbesserungen.

Das 2-stufige Verfahren sieht vor, dass Fälle, bei denen Hinweise auf eine Rehabilitations-Indikation vom pflegerischen Gutachter gesehen werden, an einen Arzt zur Entscheidung weitergeleitet werden. Fälle ohne diese Hinweise werden direkt vom Gutachter abgeschlossen. In Bezug auf die Rehabilitations-Indikation bedeutet dies jedoch eine strukturell fehlende Qualitätssicherung in diesen Fällen, da nicht geprüft wird, ob nicht irrtümlich eine falsch negative Einschätzung erfolgt ist.

Die Einschätzung zum möglichen Bestehen einer RehabilitationsIndikation ist - je nach Situation vor Ort - mit einem erhöhten Aufwand für die pflegefachlichen Gutachter verbunden. Gleichzeitig ist zum jetzigen Zeitpunkt jedoch die Zahl der Antragsteller, bei denen eine Rehabilitations-Indikation festgestellt wird, sehr niedrig. Aussagen, vor allem aus den Experteninterviews, lassen hierbei den Schluss zu, dass nicht nur die schon beschriebene zeitliche Situation, sondern auch die individuelle Einstellung der Gutachter diese Koinzidenz (mit) erklären kann. Ob das mögliche Bestehen einer Rehabilitations-Indikation immer im angemessenen und notwendigen Umfang geprüft wird, hängt demnach auch davon ab, inwieweit die Gutachter davon überzeugt sind, dass die Prüfung richtig, wichtig und der Aufwand im Verhältnis zum Ergebnis angemessen ist.

Die Ergebnisse zeigen an verschiedenen Punkten, dass es deutliche Differenzen bei den fachlichen Bewertungsstandards innerhalb und zwischen den MDK gibt. So ist der Schulungsstand der Gutachter bezogen auf MDK-übergreifende Schulungen niedriger als der Schulungsstand bezogen auf MDK-interne Schulungen. Für diese gab es jedoch bislang keine bundesweit einheitlichen Schu- lungsmaterialien, sodass sich diese Schulungen inhaltlich regional stark unterscheiden können. Da zusätzlich der Schulungsstand zwischen internen und externen Gutachtern differiert, besteht vor allem in MDK, die mit einer hohen Anzahl externer Gutachter arbeiten, ein erhöhtes Risiko für unterschiedliche Bewertungsstandards. Allerdings ergibt sich auch in Bezug auf die Bewertungsstandards der beteiligten Ärzte kein einheitlicher Eindruck. Parallel zu der geteilten medizinischen Grund- und Fachqualifikation finden sich deutlich individuelle Färbungen, die sowohl in den konkreten Reha-Entscheidungen, als auch in der Bewertungskultur der Organisationseinheit ihren Niederschlag finden. Insgesamt gingen die Einschätzungen in den Interviews und Gruppendiskussionen sowohl von Seiten der Ärzte als auch von Seiten der pflegefachlichen Gutachter stark auseinander, inwieweit die pflegefachlichen Gutachter ausreichend geschult sind oder überhaupt schulbar seien, um die Rehabilitations-Indikation angemessen einschätzen zu können ( $\triangleright$ Abb. 2).

\section{Strukturelle Unterschiede der MDK}

Die strukturellen Unterschiede der MDK, die die Steuerungswirkungen für einen GPS als Rahmenbedingungen beschränken, sind vielfältig. Sie beinhalten nicht in jedem Fall Vor- oder Nachteile für einzelne MDK, verweisen jedoch auf den Bedarf nach zum Teil individuellen Regelungen in Bezug auf Kommunikation und Entscheidungswege. Sie betreffen

- die Größe und (De-)Zentralisierung der 15 MDK,

- die organisatorische Einbindung der Ärzte in die Pflegebegutachtung,

- die Zahl der pflegefachlichen Gutachter und insbesondere das Verhältnis zwischen internen und externen, d. h. nicht festangestellten Gutachtern,

- den Fristrahmen der MDK, der mit den Pflegekassen zur Einhaltung der 5-Wochen-Frist ausgehandelt wurde, sowie

- die technische Ausstattung der MDK.

Nach den in der Strukturdatenerhebung erfassten Daten variieren die Anteile der festgestellten Rehabilitations-Indikationen, das heißt die Zahl der gestellten Rehabilitations-Indikationen bezogen auf die Zahl der erstellten Pflegegutachten nach dem 2-stufigen Verfahren zwischen den einzelnen MDK deutlich. Sie liegen in den meisten MDK für das Jahr 2012 eher niedriger als die für das Jahr 2010 vom Bundesgesundheitsministerium veröffentlichten Anteile bei Erstbegutachtungen von 1,1\% (eigene Berechnungen gemäß [7]). Ein direkter Vergleich zwischen den einzelnen MDK ist dabei 


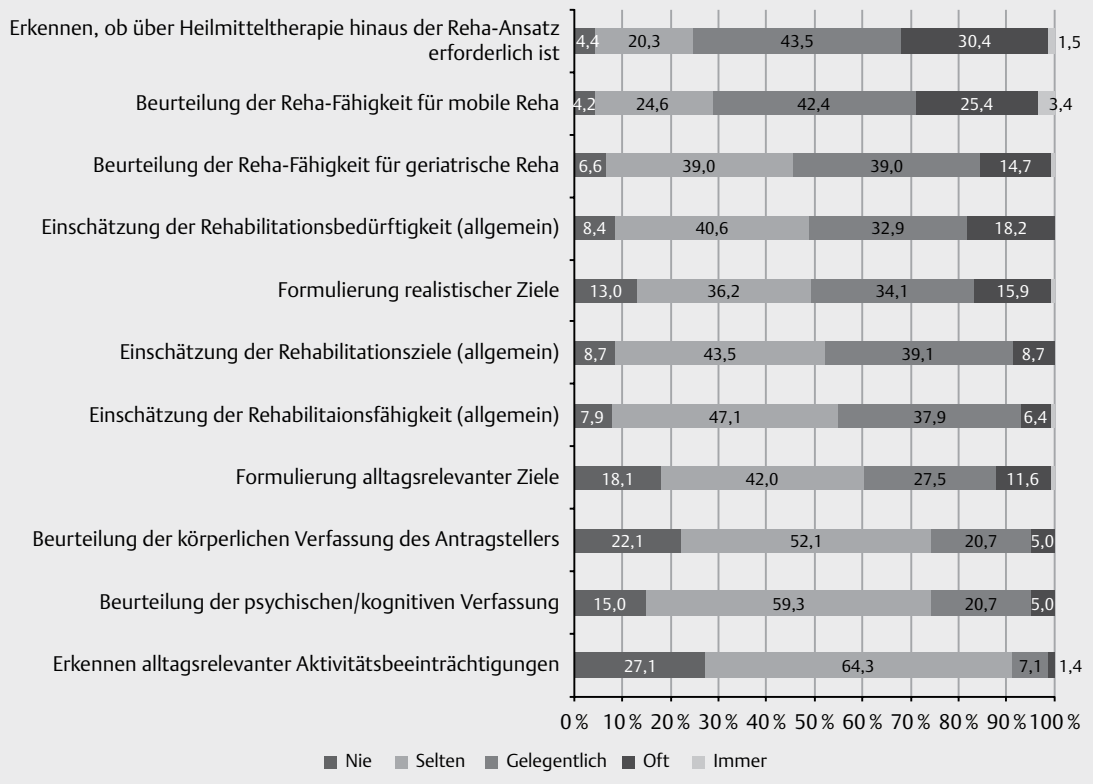

Abb. 2 Probleme der pflegefachlichen Gutachter bei der Einschätzung der Rehabilitations-Indikationskriterien. Quelle: Eigene Darstellung nach den Ergebnissen der Online-Erhebung. Anmerkung: Der Wortlaut der Frage war: „In welchen Bereichen bestehen Probleme für die Pflegefachkräfte (Gutachter) beim Ausfüllen der 3-seitigen Rehabilitationsempfehlung in Bezug auf die Rehabilitations-Indikationskriterien?"

nicht ohne weiteres möglich. Die Zahl der Gutachter, die je MDK befragt wurden, ist zu niedrig, um generalisierende Aussagen zu treffen; die Daten geben jedoch Hinweise darauf, dass die Höhe der Zahl der weitergeleiteten Gutachten sowohl aus MDK-bezogenen Gründen als auch aus personenabhängigen Gründen variiert. Die Unterschiede zwischen internen und externen Gutachtern sind ebenfalls erheblich.

\section{Heterogenität der Prozessabläufe}

Jenseits dieser grundsätzlichen strukturellen Unterschiede zeigten sich die Prozessabläufe innerhalb der MDK in einer Vielzahl von Einzelschritten als sehr heterogen. Dies betrifft zum einen formale Abläufe zum anderen - und dies ist für die Qualität der Erhebung der Rehabilitations-Indikation ein entscheidender Faktor - die Organisation der Kommunikation zwischen den pflegefachlichen und ärztlichen Gutachtern im Rahmen des 2-stufigen Verfahrens.

Unterschiede zeigten sich z. B. darin, inwieweit die 3-seitige Rehabilitations-Empfehlung vom pflegefachlichen Gutachter vorausgefüllt wurde, durch wen der Gutachtenabschluss vorgenommen wird, wie die Mitteilung über ein Gutachten mit vorliegender möglicher Rehabilitations-Indikation an den zuständigen ärztlichen Gutachter erfolgt sowie in der Erreichbarkeit der zuständigen ärztlichen Gutachter. Alternative Konzepte haben hier zum Teil sehr spezifische Vor- und Nachteile. Während z. B. der Einsatz einer zentralen ärztlichen Ansprechperson die kurzfristige Erreichbarkeit sicherstellt, ermöglicht die dezentrale Organisation über viele Ärzte den persönlichen Kontakt und erleichtert damit möglicherweise die direkte Kontaktaufnahme.

Weitere Unterschiede in den Kommunikationsabläufen zeigten sich zum einen in Bezug darauf, inwieweit die ärztlichen Gutachter die pflegefachlichen Gutachter - z. B. in strittigen Fällen - durch Rückfragen in ihre Entscheidung einbeziehen, und zum anderen dahinge- hend, ob und in welcher Form die pflegefachlichen Gutachter ein qualifiziert begründetes Feedback zu ihrer Ersteinschätzung erhalten.

Neben diesen die Kommunikation innerhalb der MDK betreffenden Faktoren, zeigen sich mit Blick auf die vorne dargestellten Schwächen 2 weitere Unterschiede: Erstens, inwieweit die Antragsteller vorab darüber informiert werden, dass die Erhebung von Informationen zur Prüfung einer möglichen Rehabilitations-Indikation Bestandteil der Pflegebegutachtung ist. Zweitens, inwieweit ergänzende Unterlagen angefordert werden, die zur Einschätzung der Rehabilitations-Indikation notwendig sein können. Gesicherte ärztliche Befunde, Entlassungsberichte stationärer Behandlungen, insbesondere vorangegangener medizinischer Rehabilitationsmaßnahmen können die Entscheidung, ob eine Rehabilitations-Indikation besteht, beeinflussen. Jedoch erfolgt seit der Einführung des PNG und der damit einhergehenden Einführung der Zusatzzahlung bei Überschreitung der Frist das Anfordern dieser Unterlagen unter zusätzlichen zeitlichen Restriktionen, da der entstehende Zeitverlust zur finanzwirksamen Überschreitung der Frist führen kann. Aus diesem Grunde wird zumindest in Einzelfällen auf die Anforderung dieser Unterlagen verzichtet. Weitgehende Einigkeit besteht darüber, dass die Entscheidung über die Rehabilitations-Indikation häufig ausschließlich auf Basis des Pflegegutachtens gefällt werden kann, auch wenn die Einschätzungen der Ärzte hinsichtlich der Validität eines solchen Vorgehens unterschiedlich sind. Da aufgrund der 5-Wochenfrist ausschließlich informelle Anforderungswege ein zeitgerechtes Eingehen von ergänzenden Unterlagen sicherstellen, ist aufgrund der regional unterschiedlichen Bedingungen eine Vereinheitlichung der Vorgehensweise in dieser Hinsicht schwierig umzusetzen. Ein häufig genannter Lösungsvorschlag in allen 3 Befragungsphasen besteht in der Forderung, fehlende Unterlagen als Entfristungsgrund anzuerkennen. 


\section{Stärken des 2-stufigen Verfahrens}

Während die bisher dargestellten Ergebnisse eine sehr kritische Bestandaufnahme des Prozesses und der Prozessabläufe nahelegen, wurden von den Gutachtern auch verschiedene Stärken des Verfahrens hervorgehoben. Zum einen ermöglicht aus Sicht vieler Gutachter die Zweistufigkeit des Verfahrens eine gute Ergänzung der pflegerischen und der medizinischen Perspektive. Durch die pflegefachlichen Gutachter erfolgt die Erhebung eines ausführlichen pflegerischen Befundes im Rahmen des Pflegegutachtens. Damit steht durch den Hausbesuch eine umfangreichere Datenbasis zur Verfügung, als dies im Regelverfahren der Rehabilitations-Begutachtung möglich ist. Die pflegerischen Gutachter erhalten einen umfassenderen Blick in die Lebensumstände des Antragstellers, als dies Ärzten gewöhnlich zur Verfügung steht. Dies belegt auch die Einschätzung der befragten Ärzte, die angaben, dass die Daten, die im Hausbesuch erhoben werden, grundsätzlich eine bessere Informationsbasis liefern können als reine Aktenlage-Gutachten. Dass für den Antragsteller nur eine Begutachtung erforderlich ist, um zum einen den pflegerischen Hilfebedarf einzuschätzen und zum anderen das mögliche Bestehen einer Rehabilitations-Indikation einzuschätzen, wurde als weitere Stärke des Verfahrens benannt. Der Antragsteller erhält zeitnah sowohl eine Pflegeeinstufung als auch eine mögliche Reha-Empfehlung. Voraussetzung für eine hohe Treffsicherheit des Verfahrens ist jedoch immer ein guter Fortbildungsstand der Gutachter.

\section{Problembereiche und Regelungsbedarfe für den GPS}

Die zentralen Problembereiche, die sich aus den hier dargestellten Schwächen des herkömmlichen Begutachtungsablaufs ergeben und für die Verbesserungen erreicht werden sollten, sind

- die Qualifikation der Gutachter und die zum Teil differierenden fachlichen Bewertungsstandards und Einstellungen,

- die Handlungssicherheit der Gutachter sowie die Kommunikation zwischen pflegefachlichen und ärztlichen Gutachtern,

- die fehlende Qualitätssicherung für negative RehabilitationsEmpfehlungen,

- negative Einflüsse durch einen erhöhten Aufwand und Zeitdruck für die pflegefachlichen Gutachter im Begutachtungsprozess und

- die Transparenz des Verfahrens für die Antragsteller.

\section{Der Gute-Praxis-Standard}

Der konsentierte Gute-Praxis-Standard beinhaltet insgesamt $7 \mathrm{Ab}$ schnitte. Während in den Abschnitten 1 und 2 Regelungen für den strukturellen Rahmen des Begutachtungsprozesses beschrieben werden, orientieren sich die Abschnitte 3-7 am Begutachtungsablauf der beteiligten Gutachter.

\section{Abschnitt 1: Schulung}

- Alle internen und externen Gutachter und beteiligten Ärzte sind standardisiert zum Thema „Rehabilitation in der Pflegebegutachtung" geschult.

- Inhalte der standardisierten Schulung sind:

1. Einschätzung der Reha-Bedürftigkeit (allgemein)

2. Erkennen, ob über die kurative Versorgung hinaus der mehrdimensionale, interdisziplinäre Behandlungsansatz einer medizinischen Reha erforderlich ist
3. Formulierung realistischer und alltagsrelevanter Ziele

4. Klare Ausschlusskriterien

5. Beurteilung der Reha-Fähigkeit für geriatrische Reha

6. Informationen zu Inhalten und Zielen von Reha-Maßnahmen

7. Diskussion von Fallbeispielen

Zusätzlich: Zusammenarbeit von Ärzten und Pflegefachkraft (u. a. Kommunikation) und Beratungskompetenz anhand des Infoflyers.

- 2-mal jährlich werden verbindliche Nachschulungen für alle Pflegegutachter durchgeführt, in denen auch Fallbeispiele zur Auffrischung und Aktualisierung des Themas Rehabilitation besprochen werden.

\section{Abschnitt 2: Unterlagen}

- Jedem Gutachter liegt ein Informationsflyer vor, in dem Informationen und Beratungsmöglichkeiten zum Thema Rehabilitation genannt sind.

- Die Gutachter haben für den Hausbesuch eine 2-seitige Handreichung, in der die wesentlichen Zustimmungs- und Ausschlusskriterien der 4 Reha-Indikationskriterien zusammengefasst sind.

\section{Abschnitt 3: Vorinformation}

- Die MDK informieren den Antragsteller schriftlich über den Termin des Hausbesuchs. In diesem Schreiben wird der Antragsteller darauf hingewiesen, dass im Rahmen der Pflegebegutachtung auch geprüft wird, ob weitere zusätzliche Maßnahmen zur besseren Bewältigung ihres Alltags, z. B. durch Maßnahmen der Rehabilitation, angeboten werden können.

- Mit gleichem Schreiben werden die Antragsteller gebeten, vorhandene Unterlagen, z. B. Facharzt-, Krankenhausberichte sowie Berichte von Rehabilitationseinrichtungen vorzuhalten.

\section{Abschnitt 4: Hausbesuch}

- Eine vollständige Prüfung der Reha-Indikationskriterien ist grundsätzlich durchzuführen. Die Abklärung der Reha-Indikation kann als abgeschlossen betrachtet werden, wenn eines dieser maßgeblichen Kriterien nicht erfüllt ist.

- Auch wenn die Bereitschaft des Antragstellers zur Teilnahme an einer Rehabilitation aktuell nicht vorliegt, sind alle Reha-Indikationskriterien vollständig zu prüfen.

- Werden im Hausbesuch Hinweise auf eine Reha-Indikation festgestellt, informiert der Gutachter situationsangemessen auf Basis der geschulten Inhalte in einem Umfang von bis zu $10 \mathrm{~min}$.

- Jedem Gutachter liegt ein Informationsflyer vor, in dem Informationen und Beratungsmöglichkeiten zum Thema Rehabilitation genannt werden. Dieser kann zur ergänzenden Information des Versicherten ausgehändigt werden.

Abschnitt 5: Nachbereitung des Hausbesuchs

- Wird ein Pflegegutachten wegen Hinweisen auf eine Reha-Indikation zur abschließenden Beurteilung an einen Arzt weitergeleitet, so ist es von Seiten des Gutachters abgeschlos- 
sen. Weitere Arbeiten oder Freigaben des Gutachters sind nicht erforderlich.

- In jedem Fall - auch bei negativer Einschätzung - wird diese Beurteilung im Gutachten nachvollziehbar begründet.

\section{Abschnitt 6: Ärztliche Entscheidung}

- Basis einer sachgerechten Entscheidung über die Reha-Indikation ist das Pflegegutachten. Dieses muss dem Arzt bei Entscheidung vollständig vorliegen.

- Sind zu einer sachgerechten Entscheidung ergänzende Informationen oder Unterlagen notwendig, werden diese durch den Arzt eingeholt. Diese Aufgabe kann auch an eigene Funktionsstellen delegiert werden.

- Sollte eine ärztliche Entscheidung nicht sofort möglich sein, kontaktiert der Arzt den Gutachter innerhalb eines Tages nach Eingang des Gutachtens (persönlich, Telefon, E-Mail) und entscheidet auf Basis dieser Beratung abschließend.

- Der Gutachter wird in jedem Fall persönlich, telefonisch oder per E-Mail über das abschließende Prüfungsergebnis und seine Begründung sowie die im Entwurf des Pflegegutachtens ggf. vorgenommenen Änderungen informiert.

- Die letztendliche Freigabe des Gutachtens erfolgt durch den Arzt.

\section{Abschnitt 7: Datenübermittlung}

- Nach Abschluss des Gutachtens werden die Daten der Rehabilitationsprüfung an die Auftrag gebende Pflegekasse übermittelt.

- Übermittelt wird das gesamte Pflegegutachten.

\section{Evaluationsergebnisse}

Für die Umsetzungsstudie und Evaluation des GPS wurden in den 7 beteiligten MDK 87 pflegefachliche und 49 ärztliche Gutachter in der Anwendung des GPS geschult und insgesamt 3247 Antragsteller nach diesem Standard begutachtet. Aus diesen Antragstellern wurden über dezidierte Verteilungsschlüssel 356 Personen ausgewählt, die in einem zweiten Hausbesuch erneut begutachtet wurden und somit in die Studienteile zu Validität und Reliabilität einbezogen wurden. 10,3\% der nach GPS durch die pflegefachlichen Gutachter erstellten Gutachten wurden dabei zur weiteren Beurteilung der Rehabilitations-Indikation an ärztliche Gutachter weitergeleitet. Im Ergebnis wurde im Mittel für 6,3 \% aller Fälle bei der Erstbegutachtung eine Indikation zur medizinischen Rehabilitation festgestellt. Dieser Wert liegt je nach MDK zwischen 3,9 und 9,9\%. In 60,8\% der Fälle, die die Pflegekräfte zur Entscheidung an die Ärzte weitergeleitet haben, haben diese dann eine Rehabilitations-Indikation festgestellt. Dies ist für ein 2-stufiges Verfahren mit einer quasi Vorfilter-Funktion und einer Entscheidungs-Funktion ein hoher Wert, der zunächst für die Qualität der pflegefachlichen Bewertungen spricht.

\section{Validität}

Zur Überprüfung der Validität der Ergebnisse der RehabilitationsEmpfehlung wurden 264 der Antragsteller durch einen ärztlichen Gutachter im Referenzverfahren ein zweites Mal begutachtet. Als zentrale Maßzahlen für die Validität des 2-stufigen Verfahrens nach GPS wurden der Positive und der Negative Prädiktive Wert sowie die Sensitivität und die Spezifität ermittelt. Die im Folgenden dargestellten Ergebnisse haben jedoch - wie bereits beschrieben - nur unter der Maßgabe Gültigkeit, dass das Referenzverfahren selber richtig diagnostiziert, was leider nicht empirisch belegt ist.

Die in $>$ Tab. 1 dargestellten Werte zeigen die Begutachtungsergebnisse der 264 Fälle, die in die Validitätsstudie einbezogen waren. Zu beachten ist, dass es sich bei diesen Fällen nicht um zufällig ausgewählte Fälle handelt, sondern um eine quotierte Stichprobe, in der Fälle mit vorliegender Rehabilitations-Indikation im 2-stufigen Verfahren nach GPS gezielt überrepräsentiert waren.

Der Negative Prädiktive Wert dieser Verteilung liegt bei 0,85 bei einem $95 \%$-Konfidenzintervall von $[0,80 ; 0,89]$. Mit einer Sicherheit von $95 \%$ kann deshalb davon ausgegangen werden, dass durch das 2-stufige Verfahren zwischen 80 und $89 \%$ der Fälle, in denen keine Rehabilitations-Indikation festgestellt wird, korrekt sind. Umgekehrt werden mit gleicher Sicherheit 11-20\% derjenigen, bei denen keine Rehabilitations-Indikation festgestellt wird, falsch klassiert.

Der entsprechende Positive Prädiktive Wert beträgt 0,67 bei einem $95 \%$-Konfidenzintervall von $[0,53 ; 0,80]$. Mit einer Sicherheit von $95 \%$ kann deshalb davon ausgegangen werden, dass durch das 2-stufige Verfahren zwischen 53 \% und 80 \% der Fälle, in denen eine Rehabilitations-Indikation ausgesprochen wird, diese Entscheidung richtig ist.

Transformiert man die quotierten Werte ( $\triangleright$ Tab. 1) auf eine zufällig gezogene Stichprobe, ergeben sich die in > Tab. 2 enthaltenen Werte. Nach diesen gilt für das 2-stufige Verfahren nach GPS ein Anteil festgestellter Rehabilitations-Indikationen von 6,3\%. Für das Referenzverfahren ergibt sich ein Anteil festgestellter Rehabilitations-Indikationen von 18,2\%. In 83,9\% der Fälle sind übereinstimmende Ergebnisse beider Verfahren zu erwarten, in 16,1 \% der Fälle abweichende.

Der Sensitivitätswert im Rahmen des Validitätstests liegt bei 0,23 bei einem $95 \%$-Konfidenzintervall von $[0,13 ; 0,37]$. Das heißt mit einer Sicherheit von $95 \%$ kann davon ausgegangen werden, dass durch das 2-stufige Verfahren zwischen 13,4 und 36,7\% der Antragsteller, die die Voraussetzungen einer Rehabilitations-Indikation erfüllen, erkannt werden. Umgekehrt heißt das, dass mit einer Sicherheit von 95\% bei 63,3-86,6\% der Antragsteller, bei denen die Voraussetzungen einer Rehabilitations-Indikation vorliegen, keine entsprechende Empfehlung ausgesprochen wird.

Der Spezifitätswert im Rahmen des Validitätstests liegt bei 0,975 , bei einem $95 \%$-Konfidenzintervall von $[0,944 ; 0,989]$. Das heißt, mit einer Sicherheit von $95 \%$ kann davon ausgegangen werden, dass zwischen 94,4 und 98,9\% der Antragsteller, die tatsächlich keine Rehabilitations-Indikation haben, auch vom optimierten Verfahren keine erhalten.

Aus diesen Werten ergibt sich zusammenfassend, dass gemessen an den Ergebnissen des Referenzverfahrens 84 \% der Einschätzungen des 2-stufigen Verfahrens richtig und $16 \%$ falsch sind. Die Abweichungen werden zu 12,7 Prozentpunkten durch ein falsch negatives Ergebnis im ersten Verfahrensschritt, zu 1,3 Prozentpunkten durch ein falsch negatives Ergebnis im zweiten Verfahrensschritt und zu 2,0 Prozentpunkten durch falsch positive Ergebnisse in beiden Verfahrensschritten verursacht. Von den eigentlich eine Rehabilitationsindikation aufweisenden Personen erhält nur etwa jeder Vierte (23,1\%) eine entsprechende Empfehlung. 
> Tab. 1 Ergebnisse der Reha-Beurteilung der Validitätsfälle nach 2-stufigem Verfahren und Referenzverfahren (absolut/ in Prozent).

\begin{tabular}{|l|l|l|l|l|l|l|l|}
\hline & \multicolumn{5}{|c|}{ Referenzverfahren } \\
\hline & & \multicolumn{3}{|c|}{ absolut } & \multicolumn{3}{c|}{ in Prozent } \\
\hline & & Reha nein & Reha ja & Gesamt & Reha nein & Reha ja & Gesamt \\
\hline \multirow{2}{*}{$\begin{array}{l}\text { 2-stufiges Verfahren } \\
\text { (GPS) }\end{array}$} & Reha nein & 188 & 33 & $\mathbf{2 2 1}$ & $71,2 \%$ & $12,5 \%$ & $\mathbf{8 3 , 7 \%}$ \\
\cline { 2 - 9 } & Reha ja & 14 & 29 & $\mathbf{4 3}$ & $5,3 \%$ & $11,0 \%$ & $\mathbf{1 6 , 3 \%}$ \\
\cline { 2 - 9 } & Gesamt & $\mathbf{2 0 2}$ & $\mathbf{6 2}$ & $\mathbf{2 6 4}$ & $\mathbf{7 6 , 5 \%}$ & $\mathbf{2 3 , 5 \%}$ & $\mathbf{1 0 0 , 0} \%$ \\
\hline
\end{tabular}

> Tab. 2 Ergebnisse der Reha-Beurteilung der Validitätsfälle nach 2-stufigem Verfahren und Referenzverfahren (absolut/ in Prozent) (Hochrechnung auf unquotierte Stichprobe).

\begin{tabular}{|l|l|l|l|l|l|c|c|}
\hline & \multicolumn{5}{|c|}{ absolut } \\
\hline & & \multicolumn{4}{|c|}{ in Prozent } \\
\hline & & Reha nein & Reha ja & Gesamt & Reha nein & Reha ja & Gesamt \\
\hline \multirow{2}{*}{$\begin{array}{l}\text { 2-stufiges Verfahren } \\
\text { (GPS) }\end{array}$} & Reha nein & 210,5 & 37,0 & $\mathbf{2 4 7 , 5}$ & $79,7 \%$ & $14,7 \%$ & $93,7 \%$ \\
\cline { 2 - 8 } & Reha ja & 5,4 & 11,1 & $\mathbf{1 6 , 5}$ & $2,0 \%$ & $4,2 \%$ & $\mathbf{6 , 3 \%}$ \\
\cline { 2 - 8 } & Gesamt & $\mathbf{2 1 5 , 9}$ & $\mathbf{4 8 , 1}$ & $\mathbf{2 6 4 , 0}$ & $\mathbf{8 1 , 8} \%$ & $\mathbf{1 8 , 2} \%$ \\
\hline
\end{tabular}

\section{Reliabilität}

Zur Überprüfung der Reliabilität der Ergebnisse der 3-seitigen Rehabilitations-Empfehlungen wurden 92 der Antragsteller durch einen zweiten pflegefachlichen Gutachter im Hausbesuch erneut begutachtet. Als zentrale Maßzahlen für die Reliabilität des 2-stufigen Verfahrens nach GPS wurden die Übereinstimmungsquoten zwischen den beiden pflegefachlichen Gutachtern ermittelt.

In 91,3\% der Fälle kamen dabei beide Pflegefachkräfte zu einem übereinstimmenden, in 8,7\% der Fälle zu einem abweichenden Urteil ( $\triangleright$ Tab. 3). Eine Auflösung nach MDK zeigt hier, dass bis auf einen Einzelfall alle Abweichungen an einem Standort produziert wurden und somit für die anderen MDK eine fast hundertprozentige Übereinstimmung bestand. Damit ist einerseits eine sehr hohe Übereinstimmung zwischen den beiden pflegefachlichen Gutachtern festzustellen, andererseits ein deutlicher Hinweis darauf gegeben, dass in einem beteiligten MDK die Standards der Beurteilung starken interindividuellen Schwankungen unterliegen könnten.

\section{Praktikabilität}

Der GPS hat in der Praktikabilitätsprüfung eine hohe Zustimmung durch die an der Studie beteiligten pflegefachlichen und ärztlichen Gutachter erfahren. Über $80 \%$ der Ärzte und über $70 \%$ der pflegefachlichen Gutachter geben an, mit dem GPS sehr gut oder gut zurechtgekommen zu sein.

Als Stärken genannt wurden vor allem die vereinheitlichte Begutachtung, die strukturierte Zusammenarbeit der beiden Gutachtergruppen und die Unterstützung durch die zusätzlichen Handreichungen, die im Abschnitt 2 des GPS benannt sind. Dies zeigt sich auch in einer deutlich erhöhten Handlungssicherheit der pflegefachlichen Gutachter, von denen fast $60 \%$ angeben, dass sich die Beurteilungssicherheit durch den GPS sehr verbessert oder verbessert habe.

Besonders positiv hervorgehoben wurden weiterhin die erfolgte gemeinsame Schulung der pflegefachlichen Gutachter und Ärzte sowie die Intensivierung der Kommunikation zwischen beiden Professionsgruppen, unter anderem auch durch das geregelte Feedback an die pflegefachlichen Gutachter. Die geäußerte Kritik am
- Tab. 3 Ergebnisse der Reha-Beurteilung der Reliabilitätsfälle durch pflegefachliche Gutachter im 2-stufigem Verfahren und nach Hausbesuch durch die zweite Pflegefachkraft (absolut/ in Prozent).

\begin{tabular}{|l|l|l|l|l|}
\hline & \multicolumn{4}{|c|}{ Pflegefachkraft 2 (Hausbesuch) } \\
\hline & & Reha nein & Reha ja & Gesamt \\
\hline \multirow{3}{*}{$\begin{array}{l}\text { Pflege- } \\
\text { fachkraft }\end{array}$} & Reha nein & 76 & 7 & $\mathbf{8 3}$ \\
\cline { 2 - 5 } 1 (Hausbe- & & $82,6 \%$ & $7,6 \%$ & $\mathbf{9 0 , 2} \%$ \\
\cline { 2 - 5 } such) & & 1 & 8 & $\mathbf{9}$ \\
& & $1,1 \%$ & $8,7 \%$ & $\mathbf{9 , 8} \%$ \\
\cline { 2 - 5 } & Gesamt ja & $\mathbf{7 7}$ & $\mathbf{1 5}$ & $\mathbf{9 2}$ \\
& & $\mathbf{8 3 , 7} \%$ & $\mathbf{1 6 , 3 \%}$ & $\mathbf{1 0 0 , 0} \%$ \\
\hline
\end{tabular}

GPS betrifft eher allgemeine Schwächen des 2-stufigen Verfahrens, die noch nicht befriedigend gelöst werden konnten. Hier sind insbesondere die nach wie vor zum Teil als nicht ausreichend beurteilte Qualifikation der pflegefachlichen Gutachter, fehlende medizinische Unterlagen und der erhöhte Zeitaufwand zu nennen. Generell sind die vorgeschlagenen Verfahrensregelungen demzufolge praktikabel, sie weisen in die richtige Richtung im Sinne eines einheitlichen und fachlich fundierten Verfahrens, es wird jedoch auch noch weiteres Optimierungspotenzial gesehen.

\section{Bewertung der Pflegekassen}

Das Ergebnis der Studie ist ein hoher Anteil positiver Rückmeldungen zu den entsprechenden Gutachten durch die PflegekassenMitarbeiter. Als problematisch in Bezug auf die Bewertung, ob die Verpflichtungen gemäß § 18a SGB XI durch die Pflegekasse erfüllt werden können, erweist sich eine Datenlücke bei der Beantwortung der entsprechenden Frage - insbesondere für Gutachten ohne Rehabilitations-Empfehlung. Die in den Gutachten in Bezug auf die 3-seitige Rehabilitations-Empfehlung dokumentierten Entscheidungen und Informationen werden jedoch zu über $80 \%$ als nachvollziehbar bewertet. Die explizit geäußerte Kritik bezieht sich in der Regel auf inkonsistent oder unvollständig ausgefüllte Gutachten. Nur in 2 Einzelfällen werden ausführlichere Informationen zur Begründung der jeweiligen Entscheidung gefordert. Optimierungsbedarf wird daher in erster Linie in Richtung einer höheren Konsis- 
tenz der ausgefüllten Gutachten und einer übersichtlicheren Übermittlungsform gesehen. Die Benennung eines direkten Ansprechpartners beim MDK wird bei $20 \%$ der bewerteten Gutachten gewünscht und könnte damit - sofern nicht bereits vorhanden eine bedenkenswerte Maßnahme sein, um Informationslücken auf schnellem und direktem Wege schließen zu können.

\section{Diskussion der Evaluationsergebnisse}

Die Ergebnisse der GPS-Umsetzungsstudie zeigen, dass bei Anwendung des GPS eine deutliche Zunahme von Indikationsstellungen zur medizinischen Rehabilitation festzustellen ist. Während der Anteil der festgestellten Rehabilitations-Indikationen bisher eher bei weniger als einem Prozent lag, wurde im Rahmen der Umsetzungsstudie ein Anteil von 6,3\% ermittelt. Für 4,2 \% der Antragsteller wurde diese Indikation auch durch das gewählte Referenzverfahren als richtig positive Einschätzung bestätigt. Der Anteilswert von 6,3 \% liegt damit deutlich über den bisher in der Praxis erzielten Werten. Das kann durchaus als Hinweis dafür angesehen werden, dass in der bisherigen Begutachtungspraxis der Rehabilitationsbedarf untererfasst wurde. Der höhere Anteil erscheint rehabilitationsmedizinisch plausibel. Die in der hier präsentierten Umsetzungsstudie festgestellten Anteile weisen in eine ähnliche Richtung, wie die in der Umsetzungsstudie zum Neuen Begutachtungsassessment (NBA) gemessenen Ergebnisse. Dort ergab sich - ebenfalls im 2-stufigen Verfahren - ein Anteil gestellter Rehabilitations-Indikationen von 4,1\%. Dabei stand die Erfassung des RehabilitationsBedarfs allerdings nicht im Mittelpunkt ([8]), die Ergebnisse wurden nicht durch ein Referenzverfahren überprüft.

Auch wenn Studieneffekte unterstellt werden können, ist doch unübersehbar, dass die Begutachtung nach GPS deutlich höhere Indikationsraten erzeugt, als das bisherige Verfahren im Regelbetrieb. Es liegt daher nahe anzunehmen, dass dafür die Anwendung des GPS ursächlich ist. Welche Elemente des GPS dabei genau zu den höheren Indikationsraten geführt haben, lässt sich nicht mit Sicherheit ableiten. Es ist wahrscheinlich, dass die zum GPS durchgeführten Schulungen hieran einen großen Anteil haben. Insbesondere ist anzunehmen, dass der professionsübergreifenden Schulung pflegefachlicher und ärztlicher Gutachter mit der Betonung der Rolle des pflegefachlichen Gutachters als vorfilternder Instanz besondere Bedeutung zukommt.

Wie einleitend festgehalten wurde, gibt es bisher keine empirisch abgesicherten Ergebnisse zur Höhe des Bedarfs an Leistungen zur medizinischen Rehabilitation bei Antragstellern der Pflegeversicherung. Auch die hier ermittelten Werte sind nicht als solche zu verstehen, da die Studie nicht darauf angelegt war. Für die Validitätsstudie wurde daher der Hausbesuch durch einen in der Rehabilitationsbegutachtung erfahrenen ärztlichen Gutachter als bestmögliches Referenzverfahren eingesetzt. Die Höhe der durch die Ärzte im Referenzverfahren festgestellten Rehabilitations-Indikationen liegt bei diesen mit einem Anteil von 18,2\% deutlich höher als zuvor erwartet. Unterstellt man ein valides Referenzverfahren, so sind die erreichten Validitätswerte - insbesondere die Sensitivität von nur 23,4\% - ausgesprochen unbefriedigend.

Die geringe Übereinstimmung der Ergebnisse der Begutachtung durch die pflegefachlichen und ärztlichen Gutachter besteht sowohl hinsichtlich falsch positiver, wie falsch negativer Beurteilun- gen und zwischen allen Begutachtungsarten. Nach diesen Ergebnissen sehen die ärztlichen Gutachter im Referenzverfahren eine Rehabilitations-Indikation also nicht nur bei mehr Versicherten, sondern offenbar auch bei anderen, als denen, die nach GPS identifiziert wurden. Überwiegend erkennen die Ärzte im Referenzverfahren bei ihrem Hausbesuch aber höhere Rehabilitationspotenziale als pflegefachliche und ärztliche Gutachter im 2-stufigen Verfahren. Dies ist insofern plausibel als die Ärzte im 2-stufigen Verfahren nur die durch den pflegefachlichen Gutachter gefilterten Informationen erhalten, aber sich nicht vor Ort selbst ein Bild machen können.

Das Ergebnis lässt 2 mögliche - sich logisch nicht ausschließende - Schlussfolgerungen zu:

1. Sofern davon ausgegangen wird, dass die Auswahl des Referenzverfahrens angemessen war und das Urteil der in der Rehabilitationsbegutachtung erfahrenen MDK-Ärzte im Hausbesuch als gültige Referenz anerkannt wird, muss das 2-stufige Verfahren nach GPS insgesamt als nicht ausreichend valide bewertet werden.

2. Angesichts der beschriebenen Limitationen des Referenzverfahrens und der festgestellten möglichen Verzerrungseffekte ist aber die Qualität und Validität der hier ermittelten Daten zu diskutieren. Hervorzuheben sind insbesondere 2 mögliche Störfaktoren: Zum einen ist es möglich, dass der Hausbesuch durch einen in der Rehabilitationsbegutachtung erfahrenen ärztlichen Gutachter, der im Rahmen dieser Studie zum ersten Mal als Referenzverfahren zur Bewertung der Rehabilitations-Indikation von Antragstellern der Pflegeversicherung eingesetzt und bisher nicht validiert wurde, für die angestrebte Prüfung weniger geeignet war, als dies von den Projektbeteiligten antizipiert wurde. Zum anderen ist zu berücksichtigen, dass zwischen dem ersten Hausbesuch im Rahmen des 2-stufigen Verfahrens und dem zweiten Hausbesuch durch den Arzt des Referenzverfahrens ein Zeitversatz von einer bis zu 4 Wochen lag. Aufgrund dieser Zeitverzögerung lag den Antragstellern zum Zeitpunkt des Hausbesuches des Referenzarztes häufig schon der Bescheid über das Ergebnis der Pflegebegutachtung vor. Die Antragsteller mussten daher die Rehabilitationsbeurteilung nicht mehr als in Konkurrenz zu ihrer Pflegeeinstufung stehend wahrnehmen. Es ist durchaus denkbar, dass die Aufhebung dieses Zielkonfliktes bei einem Teil der Antragsteller zu Verhaltensänderungen und insbesondere einer deutlich gestiegenen Motivation zur Teilnahme an rehabilitativen Leistungen geführt hat. Um entsprechende Einflussfaktoren auszuschließen, müsste das 2-stufige Verfahren zur Überprüfung auch außerhalb der Pflegebegutachtung eingesetzt und dort validiert werden. Darüber hinaus wären auch Effekte der Selbstselektion der Studienteilnehmer, durch den Zeitversatz bedingte Veränderungen des Gesundheitszustandes der Antragsteller sowie methodische Umsetzungsprobleme der Studie als mögliche Störfaktoren in Betracht zu ziehen.

Aufgrund dieser Störfaktoren wäre eine alternative Schlussfolgerung daher, dass die Aussagen zur Validität des GPS-optimierten 2-stufigen Verfahrens wenig belastbar sind.

Welche der beiden Interpretationen zutreffend ist, kann ohne weitere empirische Studien nicht abschließend beurteilt werden. Wahr- 
scheinlich ist aber, dass beide Interpretationen zumindest zum Teil zutreffend sind und es eher darum geht, zu welchen Anteilen die Ergebnisse auf Schwächen des 2-stufigen Verfahrens zurückzuführen sind und zu welchen Anteilen sie ein methodisches Artefakt sind.

Weiterhin ist zu diskutieren, inwieweit eine eindeutige richtigfalsch-Entscheidung - die die Voraussetzung für die hier angewendeten statistischen Tests auf Sensitivität und Spezifität darstellt angemessen ist. Die dabei zugrunde gelegte klar dichotomisierte Entscheidung ist sozialrechtlich gefordert und notwendig, fachlichinhaltlich jedoch in Frage zu stellen. Da es sich bei der Leistung einer medizinischen Rehabilitation um eine sehr komplexe Maßnahme handelt, ist auch die Beurteilung der Rehabilitations-Indikation ein komplexer Entscheidungsprozess, bei dem eine Vielzahl unterschiedlicher Dimensionen einbezogen werden muss. Es ist daher davon auszugehen, dass es bei dieser Entscheidung immer Spielräume geben wird, die - wenn die Prüfung auf ein dichotomes Endergebnis reduziert wird - zu Unschärfen führen müssen.

Insgesamt ist auch zu hinterfragen, ob die Entwicklung des GPS zu sehr auf strukturell-organisatorische und zu wenig auf fachlichinhaltliche Vorgaben abgestellt war. So könnte eine Erklärung für die später beobachteten inkonsistenten Begutachtungsergebnisse die nicht erfolgte professionsübergreifende Operationalisierung des Rehabilitations-Bedarfs im Rahmen des GPS sein.

Für die Reliabilitätsstudie sind die Ergebnisse allgemein positiver zu bewerten, da eine fast durchgehende Übereinstimmung der Endergebnisse vorliegt. Die deutlichen Abweichungen in einem MDK zeigen jedoch auch, dass individuell konsistente, aber interindividuell abweichende Kriterien existieren, die einer vollständigen Zuverlässigkeit entgegenstehen. Dies gilt allerdings auch allgemein für die einzelnen Items innerhalb der Prüfung, die durchweg als nicht überindividuell konsistent einzuordnen sind.

Die durchweg positiven Einschätzungen der Praktikabilitätsstudie zeigen zweierlei auf: Erstens werden die durch den GPS vorgenommenen Veränderungen wahrgenommen und weitgehend im Alltag als umsetzbar eingeschätzt. Es ist also davon auszugehen, dass die Regelungen ein gewisses Maß an positiver Wirksamkeit haben, das allerdings nach der Stärke der Veränderung variiert. In MDK, für die der GPS kaum Veränderung bedeutete, sollte die Einschätzung der Praktikabilität weniger relevant sein als in anderen MDK. Zweitens werden Art und Inhalt der Veränderung weitgehend als funktional bewertet. Vor allem die qualifikatorischen Interventionen in Form der Schulungen aller Beteiligten werden sogar noch stärker gewünscht. Hier zeigt sich eine auch schon in der ersten Projektphase geäußerte Wahrnehmung eigener Handlungsunsicherheit, die durch Bildungsmaßnahmen gezielt verringert werden kann. Allgemein werden die Veränderungen durch den GPS als Prozessschritt wahrgenommen, auf den folgend weitere Evaluationsund Anpassungsschritte notwendig sind.

Während das Verfahren hinsichtlich seiner Reliabilität und Praktikabilität vor dem Hintergrund des explorativen Zugangs zufriedenstellende Ergebnisse gezeigt hat, können über die Gültigkeit des Verfahrens im Hinblick auf die Identifikation von Versicherten mit einer Rehabilitations-Indikation keine abschließenden, wissenschaftlich abgesicherten Aussagen gemacht werden. Es zeigen sich jedoch unübersehbare Indizien für eine unbefriedigende Gültigkeit des 2-stufigen Verfahrens. Auch wenn das Ziel nur die explorative Ermittlung von Größenordnungen war, geben die unerwartet hohen Indikationsraten im Referenzverfahren und die starken Inkonsistenzen einen klaren Hinweis, dass hier ein Problem liegt. Es konnte nicht gezeigt werden, dass das GPS-optimierte 2-stufige Verfahren die Versicherten mit Rehabilitations-Indikation zutreffend identifiziert. Dieser Teil der Studie brachte somit nicht das gewünschte Ergebnis. Allerdings kann angesichts der genannten Limitationen auch nicht mit Sicherheit konstatiert werden, dass das GPS-optimierte 2-stufige Verfahren nicht valide ist.

\section{Fazit}

Die Feststellung von Rehabilitationsbedarf ist komplex, es bedarf dabei einer Abschätzung der Schwere, Komplexität und Prognose der eingetretenen oder drohenden Behinderung. Bisher existiert keine exakte Messmethode zur Feststellung des „wahren“ Rehabilitationsbedarfs. Dies gilt grundsätzlich für alle Zugangswege zu Leistungen der medizinischen Rehabilitation. Die Indikationsstellung zu Leistungen der medizinischen Rehabilitation im Rahmen der Pflegebegutachtung durch das 2-stufige Verfahren stellt somit für die pflegefachlichen und ärztlichen Gutachter eine besondere Herausforderung dar.

Vor dem Hintergrund der zunehmenden Bedeutung von Rehabilitation und anhaltender Kritik an Pflegekassen und MDK erfolgte mit dem Projekt Reha XI erstmals eine umfassende Analyse und prospektive Evaluation dieses Begutachtungsgegenstandes. Insofern handelt es sich um ein innovatives Projekt. Bisher sind keine empirischen Untersuchungen, auch nicht aus anderen Begutachtungsfeldern, mit einer vergleichbar umfassenden Datenbasis bekannt.

Wie beabsichtigt, konnte durch die Prozessanalyse der ersten Projektphase eine deutliche Erhöhung der Prozesstransparenz erreicht werden. Die hierdurch mögliche Identifikation von Stärken und Schwächen des tagtäglich verwendeten Verfahrens konnte erfolgreich als Basis einer gerichteten Prozessveränderung genutzt werden. Diese erfolgte durch den - unter Einbezug aller Beteiligten - partizipativ erarbeiteten GPS, der erstmals ein MDK-übergreifend einheitliches Verfahren für die Beurteilung rehabilitativer Bedarfe im Rahmen der Pflegebegutachtung nach SGB XI implementierte. Die Bottom-up-Strategie hat sich hierbei als für die Akzeptanz der Veränderungen äußerst gewinnbringend erwiesen. Die Vereinheitlichung des Verfahrens und die erreichte Transparenz der Abläufe bildet zugleich eine notwendige Ausgangsbedingung für die inhaltliche Überprüfbarkeit des Verfahrens. Mit dem GPS liegt nun ein empirisch gestützter Expertenkonsens vor, der standardisierte Anforderungen an den Gesamtprozess der Rehabilitations-Beurteilung im Rahmen der Pflegebegutachtung definiert.

Durch das Projekt Reha XI konnte gezeigt werden, dass die Feststellung des Reha-Bedarfs in einem 2-stufigen Verfahren grundsätzlich praktikabel und auch reliabel ist. Die durch den GPS erfolgten Optimierungen im Begutachtungsprozess haben zu einer deutlichen Qualitätsverbesserung im Begutachtungsablauf geführt und in der Folge zu mehr Rehabilitations-Indikationen.

Darüber hinaus ergeben sich aus allen Projektphasen Anknüpfungspunkte für eine weitere Qualitätsverbesserung und Fortentwicklung des GPS. Dies betrifft z. B. die inhaltliche und strukturelle Weiterentwicklung der Schulungen, die Optimierung des Gutachtenformulars sowie eine stärkere Fokussierung der Qualitätssicherung der Pflegegutachten auf nicht ausgesprochene Rehabilitationsempfehlungen. 
Es ist jedoch auch festzuhalten, dass die eingeleiteten Qualitätsverbesserungen nur im Rahmen der gegebenen rechtlichen und strukturellen Rahmenbedingungen greifen können. Ein Teil der festgestellten Schwachpunkte des Verfahrens, insbesondere Konflikte, die sich aus der Überschneidung verschiedener Leistungsbereiche des SGB ergeben, der subjektiv wahrgenommene erhöhte Zeitdruck durch die 5-Wochenfrist oder auch die grundsätzliche Organisation im Rahmen eines 2-stufigen Verfahrens können durch die Regelungen des GPS abgefedert, nicht aber grundsätzlich aufgelöst werden.

Bei der Validitätsprüfung stellte sich das grundsätzliche methodische Problem, dass es bisher kein validiertes Referenzverfahren, also keinen „Goldstandard“ gibt. Dadurch sind die Ergebnisse in ihrer Aussagekraft limitiert. Hier bedarf es auf der einen Seite weiterer rehabilitationsmedizinischer Forschung, um objektive und valide Verfahren zur Feststellung von Rehabilitationsbedarf zu entwickeln. Zum anderen sollte innerhalb der MDK die Qualität der gutachterlichen Entscheidungen weiter entwickelt und optimiert werden.

Einer objektiven und validen „Messung“ des Rehabilitationsbedarfs werden wegen der Komplexität des Prüfungsgegenstandes vermutlich immer enge Grenzen gesetzt sein. Letztlich geht es bei der Entwicklung eines entsprechenden bundesweit einheitlichen Begutachtungsstandards auch um eine Abwägung zwischen Anforderungen an die Praktikabilität auf der einen und der Qualität eines Verfahrens auf der anderen Seite.

\section{Ausblick}

Das Projekt „Reha XI“ hat wichtige Impulse für eine Weiterentwicklung der Feststellung des Rehabilitationsbedarfs in der Pflegebegutachtung - auch im Hinblick auf die anstehende Einführung des Neuen Begutachtungsassessment (NBA) - gegeben. Die MDK-Gemeinschaft hat zur Umsetzung der empfohlenen Maßnahmen das Projekt „Opti-Reha MDK (Optimierte Reha-Bedarfsfeststellung in der Pflegebegutachtung)“ im Juli 2014 initiiert.

Die im Projekt Reha XI gewonnenen Erkenntnisse wurden mit Blick auf eine flächendeckende Umsetzung in der Regelbegutachtung durch das Projektteam, bestehend aus Experten von SEG 1, SEG 2, KCG und MDS sowie ärztlichen und pflegefachlichen Anwendern des GPS der MDK, bewertet und der GPS in einen optimierten Begutachtungsstandard (OBS) überführt. Dieser orientiert sich weiterhin am praktischen Ablauf und umfasst 6 Themenbereiche von der Schulung, über Unterlagen, Vorinformationen, Hausbesuch, Nachbereitung bis zur ärztlichen Entscheidung. Ein Kernstück ist dabei ein bundeseinheitliches professionsübergreifendes Schulungscurriculum für pflegefachliche und ärztliche Gutachter. Seit Januar 2015 wird flächendeckend in allen MDK nach dem OBS begutachtet.

Mit Einführung des neuen Pflegebedürftigkeitsbegriff und des NBA im Rahmen des PSG II steht die Pflegeversicherung vor einem Paradigmenwechsel. Maßstab wird zukünftig der Grad der Selbständigkeit des Antragstellers sein, sodass bei der Begutachtung nun ein ressourcenorientierter statt defizitorientierter Ansatz verfolgt wird. Die Aufnahme eines bundeseinheitlichen strukturierten Verfahrens zur Feststellung des Rehabilitationsbedarfs (im Sinne des OBS), wie es das PSG II nun vorsieht, ist hilfreich, um eine Verstetigung der Prozessabläufe zu unterstützen. Ziel ist es, vorliegenden Rehabilitationsbedarf bei Pflege-Antragstellern so optimal wie möglich zu erkennen.
Interessenkonflikt

Die Autoren geben an, dass kein Interessenkonflikt besteht.

\section{Literatur}

[1] GKV-Spitzenverband. Bericht des GKV-Spitzenverbandes nach § 18a Abs. 3 SGBXI über die Erfahrungen der Pflegekassen mit der Umsetzung der Empfehlungen der Medizinischen Dienste der Krankenversicherung und der beauftragten unabhängigen Gutachter zur medizinischen Rehabilitation im Rahmen der Begutachtung zur Feststellung der Pflegebedürftigkeit Berichtsjahr 2013 [Internet]. 2014; Available from: https://www.gkv-spitzenverband.de/media/ dokumente/pflegeversicherung/pflege_berichte/2014_09_01_Pflege_ Bericht__18a.pdf (zugegriffen am 16.08.2015)

[2] Pick P. Geriatrische Rehabilitation. Perspektiven aus Sicht des MDK. In: Füsgen I, (Hrsg.). Geriatrische Rehabilitation. Vom Ermessen zur Pflicht - auch für den dementen Patienten, 26. Workshop des „Zukunftsforum Demenz“. Dokumentationsreihe Band 22. Wiesbaden: Medical Tribune Verlagsgesellschaft mbH; 2008: p 43-48

[3] Küpper-Nybelen J, Ihle P, Deetjen W et al. Empfehlung rehabilitativer Maßnahmen im Rahmen der Pflegebegutachtung und Umsetzung in der ambulanten Versorgung. Z Gerontol Geriatr 2006; 39: 100-108

[4] Rothgang H, Müller R, Unger R. BARMER GEK-Pflegereport 2013. Schwerpunktthema: Reha bei Pflege. Siegburg: Asgard-Verlagsservice $\mathrm{GmbH} ; 2013$

[5] Lübke N. Explorative Analyse vorliegender Evidenz zu Wirksamkeit und Nutzen von rehabilitativen Maßnahmen bei Pflegebedürftigen im Hinblick auf eine mögliche Anwendbarkeit im Rahmen der Feststellung des Rehabilitationsbedarfs bei der Pflegebegutachtung. G3-Gutachten im Auftrag des Medizinischen Dienstes des Spitzenverbandes Bund der Krankenkassen e. V. (MDS). Hamburg: Kompetenz-Centrum Geriatrie (KCG); 2015 Available from https://www.mds-ev.de/fileadmin/ dokumente/Publikationen/GKV/Rehabilitation/Gutachten_Reha_bei_ Pflegebeduerftigkeit_KCG.pdf (zugegriffen am 22.12.2015)

[6] Raspe H, Ekkernkamp M, Matthis $C$ et al. Bedarf an rehabilitativen Leistungen: Theorie und Empirie. Rehabilitation 2005; 44: 325-334

[7] Bundesministerium für Gesundheit. Bericht der Bundesregierung über die Entwicklung der Pflegeversicherung und den Stand der pflegerischen Versorgung in der Bundesrepublik Deutschland [Internet]. 2011; Available from https://www.bundesgesundheitsministerium.de/ fileadmin/dateien/Publikationen/Pflege/Berichte/Bericht_der_Bundesregierung_ueber_die_Entwicklung_der_Pflegeversicherung_und_den_ Stand_der_pflegerischen_Versorgung_in_der_Bundesrepublik_ Deutschland.pdf (zugegriffen am 22.12.2015)

[8] Windeler J, Görres S, Thomas $S$ et al. Maßnahmen zur Schaffung eines neuen Pflegebedürftigkeitsbegriffs und eines Begutachtungsinstruments zur Feststellung der Pflegebedürftigkeit. Schriftenreihe Modellprogramm zur Weiterentwicklung der Pflegeversicherung, Band 3. Berlin: GKV-Spitzenverband; 2011 